\title{
A!
}

This is an electronic reprint of the original article.

This reprint may differ from the original in pagination and typographic detail.

Österberg, Monika; Valle-Delgado, Juan Jose

\section{Surface forces in lignocellulosic systems}

Published in:

Current Opinion in Colloid and Interface Science

DOI:

10.1016/j.cocis.2016.09.005

Published: 01/02/2017

Document Version

Peer reviewed version

Published under the following license:

CC BY-NC-ND

Please cite the original version:

Österberg, M., \& Valle-Delgado, J. J. (2017). Surface forces in lignocellulosic systems. Current Opinion in Colloid and Interface Science, 27, 33-42. https://doi.org/10.1016/j.cocis.2016.09.005

This material is protected by copyright and other intellectual property rights, and duplication or sale of all or part of any of the repository collections is not permitted, except that material may be duplicated by you for your research use or educational purposes in electronic or print form. You must obtain permission for any other use. Electronic or print copies may not be offered, whether for sale or otherwise to anyone who is not an authorised user. 
Current Opinion in Colloid and Interface Science - review

\title{
Surface forces in lignocellulosic systems
}

\author{
Monika Österberg*, Juan José Valle-Delgado \\ Department of Forest Products Technology, School of Chemical Technology, Aalto University \\ P.O. Box 16300, FI-00076 Aalto, Finland
}

\begin{abstract}
Lignocellulosics, i.e., cellulose, lignin and hemicelluloses, are natural renewable polymers of high technological interest. The properties of products based on these polymers are largely determined by the forces at their interfaces. This review summarizes the main findings related to surface interactions relevant for papermaking and describes how the interest in novel, high performance renewable materials has changed the focus of the research to nanocellulosic materials. Areas of interest that need further work are also outlined.
\end{abstract}

\section{Introduction}

Lignocellulosics is a term that generally describes the main components of most plants, i.e. cellulose, hemicelluloses and lignin. Cellulose is the most abundant of these polymers. Cellulose fibres are widely used for the fabrication of paper, paper board, textiles, and composites. Hemicelluloses are important due to their role in the cell wall and the fact that they are not completely removed from the pulp fibres before their use. Hemicelluloses have also been investigated for the production of renewable composites. Lignin has traditionally been burned for energy, but there is a growing interest to find new applications for this abundant aromatic polymer. With the need to decrease our use of fossil resources, lignocellulosics are gaining a renewed interest and the research focus is shifting from traditional papermaking to e.g. development of renewable packaging materials, light weight strong composites, and materials for biomedical applications [13]. The properties of those materials are governed by the surface forces between their components. Understanding the forces in more detail is essential not only for a proper characterization of the materials, but also for the design of new functional materials and for the optimization of technological processes. 
Some of the first direct surface force measurements between cellulose surfaces were performed using the interferometric surface force apparatus (SFA) developed by Israelachvili and coworkers $[4,5]$. The SFA allows the measurement of forces between surfaces mounted in crossed-cylinder geometry as a function of their separation. The absolute value of separation is precisely determined, but transparent and smooth surfaces are required, which in practice limits the experiments to mica or modified mica substrates. The atomic force microscope (AFM) has also been extensively used to study surface forces in lignocellulosic systems. Initially developed to obtain high resolution images of substrates [6], soon the potential of the AFM for force measurements was recognized and exploited, especially after the introduction of the colloidal probe technique [7]. Typically, the interaction forces between a spherical microparticle and a flat substrate or between two spherical microparticles are measured in AFM force experiments.

In this review we summarize the main findings obtained from direct surface force measurements of lignocellulosics and describe how the research field has changed focus from papermaking to composites, biomedicals and new materials. We also give an outlook into what we envision will be future interests.

\section{Direct measurement of surface forces relevant for paper technology}

\subsection{Forces between model cellulose surfaces: effect of $\mathrm{pH}$, ionic strength, surface charge}

\section{and cellulose crystallinity}

The interest in fundamental understanding of the phenomena taking place in paper technology (papermaking, paper coating, paper recycling) has fostered many studies of surface forces in lignocellulosic systems. The first attempt to measure the interaction forces between cellulose surfaces was made by Neuman et al. in 1993 using the SFA [8•]. This was a pioneering work that included an impressive amount of systems relevant for papermaking, like cellulose in air and water, xylan in aqueous media, and sodium polyacrylate in the presence of $\mathrm{Ca}^{2+}$ ions, and was a great inspiration for future research. In their experiments, Neuman et al. used cellulose films prepared by spin-coating cellulose dissolved in trifluoroacetic acid onto mica substrates. Those films were, however, rather rough and unstable, and that affected some of their conclusions. Neuman et al. observed a very long-ranged repulsion, typical for polyelectrolyte brushes, between the cellulose surfaces in water, and they proposed a model for the cellulose surface as consisting of "molecular 
fibrils", or dangling tails, protruding into the solution. This model was not fully supported by later research using more stable and well defined cellulose substrates.

Due to the drawbacks of the cellulose substrates used by Neuman et al. [8•], two different approaches were concurrently applied to gain further understanding of the interactions in cellulosic systems. Holmberg et al. prepared model surfaces of pure, regenerated cellulose by LangmuirBlodgett (LB) deposition of trimethylsilyl cellulose on hydrophobized mica followed by desilylation in a humid $\mathrm{HCl}$ atmosphere [9••]. They extensively characterized the cellulose films with respect to wetting, topography, roughness, thickness, refractive index and chemical composition using the Wilhelmy plate method, AFM, ellipsometry and XPS. Holmberg et al. also estimated the Hamaker constant for cellulose across air or water, and those values were later confirmed by more thorough ellipsometry measurements [10]. The films were very stable and smooth, and reproducible results were obtained. In contrast to Neuman et al. the range of repulsion in aqueous media was only tens, not hundreds of nanometres. Since the range of repulsion was shorter than expected for electrostatic double layer forces at the electrolyte concentration used, they concluded that the cellulose was uncharged at the conditions of the experiments ( $\mathrm{pH}$ about 6). Simultaneously, Rutland et al. measured the surface forces between two cellulose spheres, applying for the first time the AFM colloidal probe technique to study the interaction between cellulose surfaces [11•]. They found that the forces were well described by the Derjaguin-Landau-VerweyOverbeek (DLVO) theory [12,13], with some additional steric repulsion observed at short separations, and they concluded that the cellulose surfaces were weakly charged.

The effect of the $\mathrm{pH}$ and the ionic strength on the surface forces between cellulose substrates was also studied using both SFA and AFM [14, 15••]. The larger number of surface charged groups (deprotonated carboxyl groups) upon an increase in $\mathrm{pH}$ provoked a stronger and longer range repulsion between the surfaces. An increase in ionic strength, in contrast, reduced the double layer repulsion, in agreement with DLVO theory, and screened the electrostatic interaction between the outermost cellulose chains, causing the collapse of the external dangling tails and, consequently, a reduction in the electrosteric repulsion -note that the term "electrosteric" indicates a combination of steric and double layer repulsions when charged chains from the surfaces come into contact(Figure 1) [15••]. Similar trends were observed with cellulose LB films using SFA, but steric forces dominated the interaction in that case [14]. Zauscher and Klingenberg studied the surface forces between regenerated cellulose surfaces using the colloidal probe technique and observed a similar effect of the ionic strength [16]. However, no evidence of cellulose dangling tails was observed in 
their experiments. On the other hand, Notley et al. were able to detect for the first time van der Waals attraction between cellulose surfaces at $\mathrm{pH} 3.5$, when the protonation of the carboxyl groups prevented double layer repulsion and probably decreased the swelling of the cellulose surfaces $[17 \bullet]$.

The cellulose samples used in those early experiments were weakly anionic and mainly amorphous, with the exception of LB cellulose layers and cellulose films spin-coated from Nmethylmorpholine oxide (NMMO) solution, which had a degree of crystallinity (cellulose II) around $60 \%$ [18]. The charge was due to deprotonation of carboxylic acid groups and thus $\mathrm{pH}$ dependent. Qualitatively, similar forces were observed. The smaller contact area provides better detection of weak double layer forces in AFM experiments, while steric forces seem to dominate the SFA measurements.

The force measurements set strict requirements on the cellulose substrates that can be used, while real pulp fibres have a very complex surface chemistry and morphology. Thus, much attention has been centred on developing more relevant cellulose model films as described in previous reviews $[19,20]$. The charge of pulp fibres is known to have a significant effect on dewatering and interactions with additives during papermaking. Using the AFM colloidal probe technique, Notley addressed the effect of the surface charge on the interaction between an amorphous cellulose microsphere and carboxymethylated cellulose films with different degrees of substitution of carboxyl groups on the cellulose fibres [21•]. Electrical double layer forces arose at $\mathrm{pH} 8.5$ which were higher in magnitude and longer in range as the surface charge of the cellulose films increased and the ionic strength of the solution decreased, as expected from DLVO theory. Van der Waals attraction was observed when the carboxyl groups were protonated at $\mathrm{pH} 3.5$. Using the same technique, Ahola et al. measured the surface forces between cellulose microspheres and films of cellulose nanofibrils (CNF) with low and high surface charge [22••]. The CNF films were prepared by spin-coating aqueous CNF dispersions on silica substrates using a cationic polyelectrolyte as an anchoring layer. Both steric and electrical double layer forces were observed in the interaction force curves, which were affected by the $\mathrm{pH}$, the ionic strength and the surface charge of CNF. The double layer repulsion was stronger for highly charged $\mathrm{CNF}$ at high $\mathrm{pH}$ and low salt concentration, in agreement with DLVO predictions. Changes in $\mathrm{pH}$ and salt concentration induced changes in the swelling of the film and, consequently, in steric repulsion. These were the first force measurements using CNF films. 
It is well known that crystalline and amorphous cellulose behave very differently in nature. For example, amorphous cellulose swells considerably in water, but water cannot penetrate into crystalline regions [23]. Furthermore, dissolution and regeneration of cellulose changes the crystal form from native cellulose I to the thermodynamically more stable cellulose II. Thus the scientific community has been interested in probing the effect of crystallinity on the direct forces in cellulosic systems. However, to date changes in crystallinity have been coupled with differences in charge or roughness, which has prevented drawing unambiguous conclusions about the effect of crystallinity. Notley et al. measured the surface forces between an amorphous cellulose microsphere and spincoated cellulose films of different crystallinity: cellulose I (nanocrystals), cellulose II (regenerated cellulose from NMMO solution), and amorphous cellulose (regenerated cellulose from lithium chloride/dimethylacetamide, $\mathrm{LiCl} / \mathrm{DMAc}$, solution) [24•]. Clear differences in the force curves for the different cellulose films were observed. An electrical double layer repulsion was detected for crystalline cellulose surfaces whose dependence on $\mathrm{pH}$ and salt concentration was well described by DLVO theory. Van der Waals forces at low $\mathrm{pH}$ or high ionic strength were only observed for the cellulose II sample. On the other hand, a strong steric repulsion dominated the interaction between amorphous cellulose surfaces at low $\mathrm{pH}$. However, the observed differences in the surface forces cannot be attributed only to the different crystallinity of the cellulose films because the surface charge and the surface groups of those films were also different. In fact, in a parallel work with similar experiments, Stiernstedt et al. did not observed significant differences in the force curves between cellulose surfaces of different crystallinity, all of them showing double layer and steric repulsions at long and short distances, respectively [25•].

\subsection{Effects of cationic polyelectrolytes on the forces between cellulose surfaces}

Cationic polyelectrolytes are used in the papermaking process to increase wet and dry strength as well as to retain fines and mineral filler particles on the cellulose fibres of the paper web. The interactions between anionic (fibres, fines, minerals and dissolved colloidal substances) and cationic additives during paper formation is quite complex and very much dependent on polymer type, concentration and adsorption time [26]. Usually it is challenging to achieve good strength, formation and retention simultaneously. To better understand these processes, several efforts in the literature have been devoted to analysing the effect of different cationic polyelectrolytes on the interaction between cellulose surfaces. Österberg (née Holmberg) studied how a highly charged cationic polyelectrolyte, poly[[2-(propionyloxy)ethyl]trimethylammonium chloride] (PCMA), affected the surface forces between regenerated LB cellulose films [27•]. PCMA adsorbed on the cellulose surfaces in a flat conformation, a process that reached equilibrium slowly, mainly due to 
the experimental setup [27•]. At short adsorption times $(1 \mathrm{~h})$ the adsorbed amount of PCMA was small and adhesion between the cellulose surfaces was observed when separating the surfaces. That adhesion was so strong that it led to disruption of the cellulose film, indicating that the adhesion between the cationic polyelectrolyte and cellulose was stronger than the adhesion between individual cellulose layers. However, no adhesion was detected after long PCMA adsorption time (16 h). The dependence of the interaction forces on the PCMA adsorption time suggests that nonequilibrium effects are important. As a model system for the polymer-mediated binding of mineral particles (clay, silica) to cellulose fibres in paper, Österberg et al. also studied the effect of PCMA on the interaction of LB cellulose films with mica surfaces and silica microparticles [27•, 28]. A repulsive interaction was observed between silica and cellulose surfaces before the addition of PCMA, a repulsion that could be well described by double layer forces as proved in a later work by Radtchenko et al.[29] Bridging attraction appeared when approaching cellulose and silica surfaces after the adsorption of PCMA, but PCMA also decreased the cellulose-silica adhesion upon separation [28]. On the other hand, long time PCMA adsorption overcompensated the surface charge of cellulose and mica surfaces, giving rise to repulsive interactions between the surfaces on approach and almost no adhesion on separation [27•].

In parallel experiments, Poptoshev et al. studied the effect of another cationic polyelectrolyte, polyvinylamine (PVAm), on the interaction between glass and LB cellulose surfaces using a noninterferometric surface force apparatus (MASIF) [30], observing similar overcompensation of surface charge upon polyelectrolyte adsorption [31]. The effect of different copolymers of PVAm and poly[dimethyldiallylammonium chloride] (PDADMAC) and PVAm derivatives on the adhesion between cellulose microspheres and cellulose substrates was analysed by Leporatti et al. and Notley et al., respectively $[32,33]$. Notley et al. observed that the adhesion between cellulose surfaces in the presence of a phenylboronic acid derivative of PVAm (PVAm-PBA) could be almost 30 times stronger than the adhesion induced by only PVAm, which made PVAm-PBA a very interesting polymer to enhance the adhesion between cellulose fibres in papermaking. Notley et al. also investigated the effect of $\mathrm{pH}$ on the conformation of the adsorbed polyelectrolyte. They found that at low $\mathrm{pH}$ the highly charged polymers adsorbed on the cellulose surfaces in a flat conformation and the interaction between the surfaces on approach was governed by double layer forces. The charge of the polymers decreased as the $\mathrm{pH}$ increased, which affected the conformation of the adsorbed polymer layer. At high $\mathrm{pH}$, the polymers adsorbed in a more extended conformation and steric forces dominated the interaction between the surfaces on approach. Upon retraction, the adhesion between the surfaces in the presence of PVAm-PBA was also $\mathrm{pH}$ dependent, increasing 
considerably at $\mathrm{pH}$ above 8 . It is interesting to note that there are two different approaches when studying the effect of the medium conditions on the conformation and interaction of polyelectrolyte layers: a) the medium conditions are changed before polyelectrolyte adsorption (for example, the study of the effect of $\mathrm{pH}$ in Notley et al.'s work [33]); and b) the medium conditions are changed after polyelectrolyte adsorption (for instance, the analysis of the effect of the ionic strength in Holmberg et al.'s work [28]). The conformation of the adsorbed polyelectrolyte is expected to be different in both approaches and, consequently, the surface forces as well.

The effect of the adsorption of cationic polyacrylamide (C-PAM) on the interaction between regenerated cellulose surfaces was studied by Zauscher and Klingenberg [16]. The interaction in CPAM solutions containing $0.01 \mathrm{M} \mathrm{NaCl}$ was governed by electrosteric repulsions, whose intensity and range increased as the C-PAM concentration rose. Adhesion between the cellulose surfaces was observed at low C-PAM concentrations, but the adhesion disappeared when the C-PAM concentration (and consequently the amount of C-PAM adsorbed) increased, in accordance with Österberg's findings using PCMA [27•]. Similar results were obtained when an anionic polyelectrolyte, sodium carboxymethyl cellulose (Na-CMC), was used instead of C-PAM [16]. Polyelectrolyte molecular weight and charge density also play an important role on the interaction between cellulose surfaces, as revealed by Salmi et al.[34•]. The surface forces between two cellulose microspheres in the presence of C-PAM and PDADMAC of different molecular weights and charge densities showed: $i$ ) a main contribution of double layer forces in the case of highly charged polyelectrolytes, which tended to adsorb on the cellulose surfaces in a flat conformation; and $i$ ) a main contribution of steric forces at high concentrations of low charged, high molecular weight polyelectrolytes, which adsorbed on the cellulose surfaces in a more expanded conformation (Figure 2). Salmi et al. extended their research to analyse the effect of cationic polyelectrolyte complexes formed by the combination of cationic and anionic polyacrylamides (C-PAM and APAM) in a mass ratio of 10:1 [35••]. The adsorption of cationic polyelectrolyte complexes on cellulose microspheres gave rise to strong, long range steric repulsions between the cellulose surfaces on approach and long range pull-off forces on retraction, an effect that intensified as the size of the complexes increased. Those characteristics of the force curves could explain the improvements in flocculation, dewatering and paper strength observed with cationic polyelectrolyte complexes compared to single polyelectrolytes in the papermaking process.

In general, the surface force measurements described above correlates well with the understanding of interactions between cellulose fibres and polyelectrolytes obtained from practical 
experience and macroscopic systems. The timescales and hydrodynamics of real papermaking cannot be mimicked in force experiments but, nevertheless, the force measurements confirm that low surface coverage and extended conformation of polyelectrolytes are important for efficient retention of fines and filler. In papermaking, three flocculation mechanisms are suggested: patch flocculation between polymer covered and uncovered patches, charge neutralisation, and polymer bridging. Force measurement results from several reports indicated that polymer-mediated binding is an important flocculation mechanism, probably more important than charge neutralization, but it is not straightforward to distinguish between polymer bridging and patch flocculation.

The research on surface forces between cellulose surfaces with or without polymers in aqueous media described here has enhanced our understanding of forces present during the formation of the paper sheet. However, it must be noted that the force experiments in aqueous solutions do not provide relevant information about paper strength, because that strength involves other mechanisms taking place during the drying of the paper. Strong adhesion between cellulose surfaces in aqueous media does not necessarily correlate with stronger paper; instead, it could lead to fibre flocculation and improper formation of paper. Force experiments during the drying of the cellulose system would be needed to characterize the mechanisms responsible for paper strength, but to carry out such experiments is very challenging.

\subsection{Friction forces between cellulose surfaces}

Together with normal surface forces, lateral (friction) forces between cellulose fibres play a key role in papermaking because they affect the rheological characteristics of the pulp suspension, the homogenization and casting of the pulp during the paper formation, and the mechanical properties of the final paper. Therefore, it is not surprising that special attention has been paid to the study of the friction forces between cellulose surfaces. Amelina et al. analysed the friction forces between crossed individual cellulose fibres in air, in water, and in aqueous solutions of a cationic polyelectrolyte (polyethyleneimine, PEI) and cationic surfactants (tetrabuthylammonium iodide, TBAI, and cetyltrimethylammonium bromide, CTAB) using a home-made apparatus [36-38]. Cellulose fibres from different origin and subjected to different treatments, with diameters in the range 10-30 $\mu \mathrm{m}$, were used in the experiments. The friction coefficient between the cellulose fibres depended on the concentration of PEI or surfactant in solution, showing a maximum around the point where PEI or surfactant adsorption neutralized the negative surface charge of the fibres. 
The potential of the AFM and the colloidal probe technique for high-sensitivity measurements of friction forces has been exploited to study the friction between cellulose surfaces at micronanoscale. Huang et al. measured the friction forces between two cellulose fibres, one of them (a fibre fragment) attached on an AFM cantilever [39]. The experiments, accomplished in air at 35$45 \%$ relative humidity, showed that the friction coefficient increased as the fibre surface became rougher. However, the adhesion between the fibres -inferred from the intersection of the frictionversus-load curves with the $\mathrm{Y}$ axis- decreased as the surface roughness increased.

The effect of the relative humidity on the adhesion and the friction forces between cellulose surfaces was studied by Feiler et al. [40•]. Both the adhesion and the friction forces between two cellulose microspheres were observed to increase significantly at relative humidities above 55\%. The friction hysteresis, that is, the separation between the loading and unloading sections of the friction curves, also increased with the relative humidity. The results were explained in terms of the formation of water capillary condensates between the contacting asperities of the rough cellulose surfaces. This was well in line with earlier findings by Holmberg et al. of capillary condensate formation around cellulose surfaces in contact in humid air [9••]. Feiler et al. pointed out that the threshold at 55\% relative humidity correlated well with the onset of paper weakening, suggesting that water capillary condensates on the surface of fibres could be responsible for that. In an earlier work, Garoff and Zauscher studied the adhesion and friction between chemically functionalized AFM tips and regenerated cellulose films at $8 \%$ and $40 \%$ relative humidity [41]. Their experimental data showed that the adhesion and the friction were higher at $40 \%$ relative humidity, which was ascribed to capillary condensation. Both adhesion and friction decreased after coating the cellulose surface with monolayers of different fatty acids. Lower values of adhesion and friction forces and a more negligible effect of the humidity were observed as the hydrophobicity of the coated layer increased, in line with a lower tendency for the formation of water capillary condensates on the surfaces. On the other hand, Bogdanovic et al. studied the friction between a cellulose microsphere and silica surfaces modified either by adsorbing alkylketene dimer (AKD, a commercial sizing agent used for paper hydrophobization) or by grafting a layer of methyl groups [42]. The experiments, carried out in air at $35-40 \%$ relative humidity, revealed that the friction forces and friction coefficients between cellulose and silica surfaces decreased in the order: bare silica > AKDcoated silica $>$ methylated silica.

The AFM colloidal probe technique has also been used to study the friction between cellulose surfaces in aqueous media. Stiernstedt et al. analysed the effect of the cellulose nature on the 
friction forces between cellulose surfaces in $0.1 \mathrm{mM} \mathrm{NaCl}$ solution [25•]. They observed a linear correlation between surface roughness and friction coefficient, qualitatively in line with Huang et al.'s observations in air mentioned above [39]. On the other hand, Zauscher and Klingenberg measured the friction forces between regenerated cellulose surfaces in the absence and presence of cationic and anionic polyelectrolytes (C-PAM and $\mathrm{Na}-\mathrm{CMC}$, respectively) [43•]. The friction forces and the friction coefficients clearly decreased as the polyelectrolyte concentration in solution increased, in good accordance with the stronger repulsion observed between the surfaces as the adsorbed amount of polyelectrolyte increased.

Deinking -the removal of ink particles from cellulose fibres- is an important step in the recycling of waste paper. Surfactants are used to facilitate the detachment and separation of ink particles from the pulp during the recycling process. Theander et al. aimed to gain a better understanding of the interactions between cellulose and ink using the AFM colloidal probe technique $[44,45 \bullet \cdot$. They studied the normal and friction forces between a cellulose microsphere and an alkyd resin substrate (a model ink surface) in surfactant solutions at different concentrations. Nonionic (hexa-ethyleneglycol mono n-dodecyl ether) and anionic (sodium oleate) surfactants frequently utilized in the deinking process were selected for the experiments. A small adhesion was detected between cellulose and alkyd resin, but it disappeared in the presence of surfactant. An important reduction in friction forces and friction coefficients was also observed as the surfactant concentration in solution increased (Figure 3). The reduction in both adhesion and friction between cellulose and alkyd resin explains why surfactants favour the detachment of ink from pulp under mechanical shear.

\subsection{Surface forces in other lignocellulosic systems}

Since cellulose is the main component in the plant cell wall, most research has focused on cellulose interactions. However, lignin and hemicelluloses play an important role in the plant cell wall. Hemicelluloses are present in varying amounts on the surface of all pulp fibres and are known to affect the water binding ability of fibres and the paper strength. The surface of pulp fibres from mechanical pulping processes may also be almost fully covered by lignin. In spite of that, the interactions in the presence of lignin has gained only limited attention. Using the colloidal probe technique, Notley and Norgren studied the interaction between a cellulose microsphere and lignin films spin-coated from kraft lignin solutions in $0.75 \mathrm{M}$ ammonium hydroxide on silica substrates [46••]. Repulsive double layer forces were typically observed at different $\mathrm{pHs}$ and ionic strengths in agreement with DLVO theory. The double layer repulsion was stronger as the $\mathrm{pH}$ increased, 
indicating that the surface charge was $\mathrm{pH}$ dependent. Steric forces at short distances were especially evident at high $\mathrm{pH}$, when the lignin film swelled.

More attention has been devoted to the interaction between hemicelluloses (mainly xylan and xyloglucan) adsorbed on cellulose or other model surfaces. The pioneering research of Neuman et al. [8•] on surface forces between xylan-coated mica surfaces was extended by Österberg et al. to analyse the effect of the charge density of xylan and the ionic strength of the solution [47]. The force curves showed an electrosteric repulsion between xylan-coated mica surfaces, stronger for the xylan with the highest charge density due to its adsorption on mica in a more extended conformation. The experimental data were fit satisfactorily to a model for the compression of anchored polyelectrolytes. As the ionic strength of the solution increased, the adsorbed layers of xylan adopted a more compact conformation and the electrosteric repulsion decreased both in magnitude and range. Force experiments between xylan-coated cellulose surfaces were carried out by Paananen et al. [48•]. Xylan adsorbed slowly and irreversibly on the cellulose surfaces (two cellulose microspheres), increasing the repulsion -double layer and electrosteric forces- between the surfaces as the xylan concentration in solution increased. The adhesion between the cellulose surfaces was also observed to decrease upon the adsorption of xylan. The weak or negligible adhesion between the xylan-coated cellulose surfaces seems to be in contradiction with previous works indicating that the adsorption of xylan increased paper strength. Paananen et al. suggested that the xylan-mediated strengthening of paper must be due to effects of xylan on the fibre bonds during the drying of paper.

Besides the negatively charged xylan, a non-charged hemicellulose, xyloglucan, has also been the subject of important research. The effect of xyloglucan on the interaction between cellulose surfaces was studied by Stiernstedt et al. [25•, 49••]. Xyloglucan adsorbed strongly to cellulose, increasing the repulsion between the cellulose surfaces on approach and the adhesion on separation, probably due to bridging [49••]. The friction forces were significantly reduced after xyloglucan adsorption, although the absolute values of the friction coefficients depended on the roughness of the cellulose surfaces as well $[25 \bullet, 49 \bullet \bullet]$. The low friction combined with higher adhesion indicates that xyloglucan could be a good additive in papermaking, although the price of xyloglucan may restrict its use in practice. Further research on surface forces between cellulose and xyloglucan was carried out by Nordgren et al.[50]. They studied the friction between a cellulose microsphere and a thiolated xyloglucan layer grafted to a gold substrate. The exposure of the xyloglucan layer to enzymatic digestion by the endo-xyloglucanase TmNXG1 increased both the surface roughness of 
the layer and, consequently, the friction forces and friction coefficients. A considerable adhesion between cellulose and the grafted xyloglucan layer was also observed in normal force curves.

The interaction between cellulose and chitosan, a natural cationic polymer, has also been investigated. Holmberg et al. measured the surface forces between a LB cellulose film and a chitosan layer adsorbed on mica, observing a long range attraction probably due to bridging when the surfaces approached each other in $0.1 \mathrm{mM} \mathrm{KBr}$ solution at $\mathrm{pH}$ about 6 [9••]. A more exhaustive study of the surface and friction forces between chitosan-coated cellulose surfaces in aqueous solutions at different $\mathrm{pH}$ was carried out by Nordgren et al. [51]. In agreement with previous investigations, the interaction between two neat cellulose microspheres showed a combination of double layer and steric repulsions at high $\mathrm{pH}$, but only steric repulsion was observed at low $\mathrm{pH}$ due to the protonation of the surface carboxyl groups. The adsorption of chitosan on the cellulose microspheres completely changed the $\mathrm{pH}$-dependence of the interactions on approach: a long range electrosteric repulsion appeared at low $\mathrm{pH}$ due to the extension of the charged chains of chitosan, whereas at high $\mathrm{pH}$, when the uncharged chains of chitosan collapsed, a shorter range steric repulsion was observed. The adsorption of chitosan also increased the adhesion between the cellulose microspheres on separation. Furthermore, a reduction in friction between the cellulose surfaces was observed upon chitosan adsorption, especially evident at low $\mathrm{pH}$.

\section{Direct surface force measurements as a tool in the development of novel materials from lignocellulosics}

\subsection{Novel lignocellulosic materials}

A growing interest in the development of novel lignocellulose-based materials for new applications has arisen in the last years $[1,52]$. Composites, antimicrobial materials, optical sensors, conductive materials, adhesives, and biomedical devices are some examples of the new utilization of lignocellulosic systems. The analysis of the surface forces between the components of those materials can provide very useful information not only to understand the material characteristics and performance, but also to support the design of new materials with special properties.

Proteins with a special affinity for cellulose are interesting systems with potential for new biotechnological applications. Cellulose binding domain (CBD) peptides have been studied as agents to induce adhesion between cellulose surfaces. Nigmatullin et al. used the colloidal probe 
technique to measure the interaction between cellulose microspheres and cellulose substrates in the presence of CBD and a recombinant protein containing two CBDs (HFBI-CBD) [53]. The attachment of CBD reduced the double layer repulsion between the cellulose surfaces, but only a small adhesion was observed at high ionic strength. In contrast, HFBI-CBD induced a significant adhesion between the surfaces, probably due to the formation of crosslinks through the two CBDs. As in the case of cationic polyelectrolytes, an excess of HFBI-CBD caused a reduction in adhesion because the accessibility of CBDs to the cellulose surfaces was sterically impeded. On the other hand, the affinity of the enzyme cellulase for cellulose and lignin has been analysed by Qin et al. through adhesion experiments at $\mathrm{pH} 4.8$ [54]. Those results shed some light on the phenomenon of lignin-mediated inhibition of the enzymatic conversion of cellulose to glucose in lignocellulose biomass for biofuel production. Similar experiments were carried out by Arslan et al., who measured the adhesion of a model cellulase (carbohydrate-binding module) attached on AFM cantilevers to different lignocellulosic substrates with controlled composition [55].

Nanocellulose, both in the form of long and flexible cellulose nanofibrils (CNF) (also called nanofibrillated cellulose, NFC) and rod-like cellulose nanocrystals (CNC) (also called nanocrystalline cellulose, NCC), is by far the lignocellulosic material that has currently gained the most attention [52]. CNF are fibrils of 5-60 nm in diameter and up to several micrometres in length obtained by the mechanical fibrillation of cellulose fibres, sometimes assisted by chemical or enzymatic pretreatments. CNF contain native cellulose I (degree of crystallinity around 60\%), amorphous cellulose and hemicellulose. Unmodified CNF is weakly charged, but the charge can be tuned by chemical pretreatments. CNC are highly crystalline cellulose rods of 5-70 $\mathrm{nm}$ in diameter and 100-250 $\mathrm{nm}$ in length obtained by acid hydrolysis of cellulose pulp. The degree of crystallinity is above $80 \%$ and no hemicelluloses are present on the surface. Ahola et al. were the first ones to prepare CNF films suitable for direct surface force measurements, as discussed previously in this review [22••]. The CNF fibrils, prepared without chemical pretreatment and containing 20-30\% hemicelluloses, may well be the most relevant model for the bleached kraft pulp fibre surface. CNC films were also used early on as a model cellulose substrate due to the high degree of crystallinity, although the sulphate groups on the surfaces renders the substrates more anionic than native cellulose [24•].

\subsection{Surface forces in cellulose-based multilayer materials}

The formation of multilayers by the sequential adsorption of polyelectrolytes of opposite charge (layer-by-layer deposition) is an interesting technique to develop materials with tailored properties. 
Sensors, antireflective coatings, drug delivery systems, biofouling agents, and functional coatings are some possible applications of polyelectrolyte multilayers. The possibility of exchanging one or both (anionic and cationic) polyelectrolytes with solid nanoparticles has inspired research on the interactions within these layers and interesting effects of both the surface charge and the structure of the components were found. Salmi et al.[56], Aulin et al. [57], Eronen et al. [58••], and Olzewska et al.[59] investigated the surface forces during the in situ build-up of different multilayers, whereas Cranston et al. studied the interactions between preformed multilayers and modified colloidal probes $[60 \bullet \bullet$.

Salmi et al. compared the forces between multilayers formed by the cationic polyelectrolyte CPAM and either silica nanoparticles or CNF as the anionic component [56]. They found that the small spherical silica nanoparticles could migrate into the C-PAM layers, whereas the stiffer CNF fibrils formed individual layers. They attributed these phenomena mainly to the difference in size and shape but also to the tendency of CNF to form networks. Eronen et al. demonstrated that it is possible to build multilayers using $\mathrm{CNF}$ as the anionic component and cationic $\mathrm{CNF}$ (formed by the reaction of $\mathrm{CNF}$ with epoxytrimethylammonium chloride), cationic starch or PDADMAC as the cationic counterpart [58••]. The surface charge of CNF and the type of cationic counterpart affected the dynamics of multilayer formation, as well as the amount adsorbed and the conformation of the multilayers. Force experiments were carried out to probe the surface forces during the in situ multilayer build-up on both a highly charged CNF film and a cellulose microsphere. Electrosteric repulsions dominated the interaction between the surfaces on approach at any stage of the multilayer formation, but different trends were observed when the cationic compound was either cationic CNF or PDADMAC. The adsorption of highly charged PDADMAC provoked deswelling of the CNF film underneath (confirmed in adsorption experiments by quartz crystal microbalance with dissipation, QCM-D) and a reduction in the electrosteric repulsion (Figure 4a). The adsorption of cationic CNF, in contrast, did not compress the CNF films underneath, giving rise to a higher, more long-ranged repulsion between the surfaces (Figure $4 \mathrm{~b}$ ). The adhesion between the surfaces was markedly stronger when the anionic CNF was the topmost layer. The QCM-D results showed that non-electrostatic forces were important for the interactions between anionic and cationic CNF.

Cranston et al. studied the formation of multilayers of $\mathrm{CNC}$ and poly[allylamine hydrochloride] (PAH) and analysed the surface forces between the preformed multilayers and spherical, functionalized silica colloid probes in aqueous solutions at different $\mathrm{pHs}$ and ionic strengths [60••]. In general, the interaction was dominated by double layer forces, repulsive between charged 
surfaces of the same sign and attractive between oppositely charged surfaces. As predicted by DLVO theory, the double layer forces were screened at high ionic strength. However, a non-DLVO attraction between the surfaces was observed at low $\mathrm{pH}$, which became stronger as the ionic strength increased (Figure 5). The authors considered that the swelling of the PAH/CNC film at low $\mathrm{pH}$ allowed highly charged PAH chains to extend into solution despite the uppermost layer being $\mathrm{CNC}$. Therefore, the attraction observed at low $\mathrm{pH}$ was attributed to polymer bridging enhanced by double layer attraction between the oppositely charged $\mathrm{PAH}$ chains and the bare or $\mathrm{COOH}-$ functionalized silica probes.

The formation of all-cellulose multilayers via layer-by-layer deposition of CNC and cationic CNF was characterized by Olszewska et al. [59]. Force experiments between the multilayers and cellulose microspheres were conducted in aqueous solutions. The build-up of the multilayers and the surface forces were affected by the $\mathrm{pH}$, as expected from the $\mathrm{pH}$ dependence of the protonation state of surface carboxyl and amine groups. The interaction between multilayers and cellulose probes was attractive at $\mathrm{pH} 4.5$ (stronger attraction when the cationic CNF was the capping layer) and repulsive at $\mathrm{pH} 8.3$ (more repulsive when $\mathrm{CNC}$ was the topmost layer). The comparison of the force curves with those obtained for single layers revealed that not only the top layer, but also the underlying layer affected the surface forces measured from the multilayers, in line with previous findings $[60 \bullet \bullet$.

\subsection{Surface forces in cellulose-based composite materials}

Currently there is a great interest in developing strong, stiff, tough and lightweight composite materials from $\mathrm{CNF}[3,61]$. The research is inspired by the extraordinary strength and toughness of natural materials like spider silk and nacre. In these natural materials the main component is the loadbearing hard material that is "glued" together by thin layers of soft polymers in highly ordered structures. The soft layer acts as an energy-dissipating material, hindering crack propagation. The toughening mechanisms of the soft polymer are complex and may include lubrication, weak links, sacrificial bonds, partial pull-out of reinforcing components and conformational changes [62-65]. In spite of the interest in characterizing and mimicking these materials, very few attempts to apply direct surface force measurements to probe the interactions within biomimetic composites have been reported. Olszewska et al. studied the effect of the physical adsorption of polyethylene glycol grafted carboxymethyl cellulose (CMC-g-PEG) on the friction and surface forces of CNF in both air and aqueous media $[66 \bullet, 67 \bullet]$. With those experiments they tried to understand the interactions responsible for the toughness during composite formation as well as within a dry composite. They 
found a pH-dependent, long-ranged electrosteric repulsion between cellulose surfaces after adsorption of CMC-g-PEG. The repulsion was much longer in range than the repulsion due to the adsorption of plain CMC. That repulsion considerably reduced the friction forces between the cellulose surfaces. The lubrication of CMC-g-PEG was $\mathrm{pH}$ dependent, with up to an $88 \%$ reduction in the friction coefficient with respect to the bare cellulose surfaces at $\mathrm{pH}$ 7.3. The lubrication provided by CMC-g-PEG facilitates the formation of even dispersions of CNF, which leads to a composite with less flocs and defects. In order to gain insight into the interactions between the fibrils within the dry composite, similar force experiments between CNF films and cellulose microspheres with or without adsorbed CMC-g-PEG were carried out in air (35\% relative humidity) by the same team [67•]. Van der Waals attractions were observed between all the surfaces on approach, with a Hamaker constant lower than expected because of the roughness of the surfaces. A strong adhesion between the cellulose surfaces was observed on retraction, which was reduced -but not cancelled- after the adsorption of CMC-g-PEG on any of the surfaces. High friction forces were measured for CNF films, with a clear hysteresis in the friction-versus-load curves due to partial pull-out of fibrils when decreasing the applied load. The adsorption of CMC-g-PEG on CNF films prevented that frictional hysteresis and significantly reduced the friction forces and friction coefficients. Moderate adhesion and low friction coefficients between cellulose fibrils coated with CMC-g-PEG could explain the improved mechanical properties of free standing CNF/CMC-g-PEG composite films, which were stronger and tougher (more plastic deformation) than CNF films [67•]. The lubrication both in air and in aqueous media is important in biomimetic composites. As an example, it has been shown -without explicit force measurements- that a lubricating PEG layer facilitates the orientation of fibrils during stretching, thus increasing the strength of composites [68]. More research on the direct measurement of surface forces is needed to completely characterize the interactions in biomimetic composites.

Cellulose fibers and fibrils are also used to reinforce hydrophobic polymer matrixes. In these applications one of the main limiting factors hampering the performance of the materials is the compatibility with the surrounding polymer matrix. Acknowledging the importance of adhesion for the final properties of (nano)composites, Lahiji et al. carried out a basic study on the effect of CNC nature on the adhesion to a silicon tip of an AFM cantilever [69]. The experimental data, obtained under ambient conditions at $20 \%$ relative humidity, revealed differences in adhesion properties that were ascribed to differences in surface composition due to the cellulose source, or alternatively, variation in the contact area due to differences in the diameter of the crystals. 
Grafting polymer chains to cellulose may be an efficient method to enhance compatibility as shown by Nordgren et al. [70]. They characterized the compatibility of cellulose with poly[ $\varepsilon-$ caprolactone] (PCL), a promising polymer for biocomposite applications, using the colloidal probe technique. The adhesion between two cellulose microspheres, with and without PCL grafted on the surface, was measured in air at $20^{\circ} \mathrm{C}$ and $60^{\circ} \mathrm{C}$. A conventional dispersion adhesion was observed between a neat cellulose sphere and a PCL-grafted cellulose sphere, suggesting no intrinsic affinity of PCL for cellulose. However, two PCL-grafted cellulose spheres showed strong adhesion with a gradual detachment over a long separation range, a phenomenon that was more prominent at high temperature and at longer contact times between the surfaces. The authors ascribed that adhesion to a diffusion-based interpenetration of the PCL layers during contact, and pointed out that nanocomposites based on PCL-grafted cellulose particles will be stronger and able to resist local detachment of the matrix during flexing, creep or shocking. Similarly, Guan et al. grafted guanidine polymer -a polymer with antimicrobial properties- on cellulose fibres [71]. The attraction and the adhesion between a spherical borosilicate particle and cellulose fibres with and without grafted guanidine polymer were studied in air. $\mathrm{CNC}$ grafted with a thermoresponsive polymer, poly[Nisopropylacrylamide] (poly(NiPAAm)), were prepared and characterized by Zoppe et al [72]. Force measurements between thin films of $\mathrm{CNC}$ and colloidal silica probes were conducted in $\mathrm{NaCl}$ solutions. While a double layer repulsion was observed in the force curves with unmodified CNC, steric forces dominated the interaction of poly(NiPAAm)-grafted CNC. An increase in $\mathrm{NaCl}$ concentration caused a collapse of the grafted polymer brushes and reduced the steric repulsion. The adhesion between silica and poly(NiPAAm)-grafted CNC also decreased upon increasing the ionic strength.

\subsection{Surface forces in cellulosic materials for biomedical applications}

In addition to renewable composites, today there is also a special interest in biomedical applications of lignocellulosics [2]. Greene et al. developed a cartilage-inspired lubrication system based on a reconstituted cellulose fiber network with adsorbed CMC and CMC-g-PEG [73]. Friction experiments of their system against stainless steel surfaces were accomplished in phosphate buffered saline (PBS) with a pin-on-disk tribometer. Lower friction coefficients were obtained as the amount of CMC in the material increased and when CMC-g-PEG was present in the PBS solution. A friction coefficient close to the one measured for a cartilage tissue was obtained at $2.5 \%$ wt CMC, in PBS with $1 \%$ wt CMC-g-PEG. The friction coefficient was also observed to decrease as the shear velocity of the experiments increased. 
With the aim of developing cellulose-based lubricating films with potential applications for cartilage implants, Valle-Delgado et al. studied the lubricating properties of CNF films with hyaluronic acid (HA), a natural component of cartilage and synovial fluid in joints [74•]. HA was attached to CNF films via an esterification reaction between the carboxyl and hydroxyl groups. The AFM colloidal probe technique was used to measure the surface and friction forces between glass microspheres and CNF films with and without $\mathrm{HA}$ in buffers at different $\mathrm{pH}$ and ionic strength. A strong electrosteric repulsion was observed after attaching HA to CNF films (Figure 6a). The magnitude and range of that repulsion increased with the swelling of the attached HA layer, reaching maximum values at high $\mathrm{pH}$ and low ionic strength. The attachment of HA considerably improved the lubrication properties of CNF films (Figure 6b). A remarkable reduction in friction forces and friction coefficients was observed at high $\mathrm{pH}$ and low ionic strength, conditions that favoured the swelling of the attached HA layer. Friction coefficients of the same order of magnitude as those for articular cartilage were obtained, suggesting that CNF-HA could be a promising material for cartilage implants, where the exceptional lubricating properties of HA are combined with the excellent mechanical properties of CNF.

\section{Conclusions}

The SFA and the AFM colloidal probe techniques have proved to be very useful for the direct measurement of surface forces in lignocellulosic systems, providing essential information on interactions at micro and nanoscale that is crucial for a proper understanding of macroscopic material properties. The surface and friction forces between different cellulose surfaces have been extensively probed in different conditions. In general, the interaction in aqueous media between two cellulose surfaces is dominated by repulsive double-layer and steric forces, whose magnitude and range depends on the roughness and surface charge of the cellulose substrates, and on the $\mathrm{pH}$ and the ionic strength of the media. Van der Waals forces were observed when those repulsive forces were successfully screened at low $\mathrm{pH}$. The effect of cellulose crystallinity, however, is still unclear.

The measurement of surface and friction forces in cellulose systems with the AFM colloidal probe technique has allowed the fundamental characterization of different phenomena in papermaking, like the interaction between cellulose surfaces and cationic polyelectrolytes (including polyelectrolyte complexes or multilayers), the deinking process, the lubrication induced by polyelectrolytes, the effect of hydrophobic coatings, and the influence of the relative humidity on 
the formation of water capillary condensates. Hemicellulose-coated surfaces have also been studied to some extent, but not much attention has been paid to lignin substrates. Currently, there is a renewed interest in finding novel applications for lignin, a major side stream of modern biorefineries. Thus, the study of surface forces in lignin systems may arise in the future.

The development of novel lignocellulose-based materials has become a hot topic during the last years. Cellulose nanofibrils and nanocrystals are considered very promising elements for new nanocomposites, functional coatings, sensors or cell culturing scaffolds. The grafting or physical adsorption of polymers has been shown to be a useful approach to provide cellulose substrates with antimicrobial, thermoresponsive or lubricating properties, as well as to improve their compatibility with the polymer matrix in nanocomposites. It is envisioned that new studies on the surface forces in nanocellulose systems will be carried out in the future, since there are still open questions. The direction of the forthcoming research will depend on the most promising applications. In addition to biomimetic composites, the increasing interest in biomedical applications of nanocellulose will probably inspire new investigations on the interaction of cellulose with, for example, proteins or even, living cells.

\section{Acknowledgements}

MÖ acknowledges the Academy of Finland for financial support (project number 278279, MIMEGEL).

\section{References}

[1] Wegner TH, Jones EP. A fundamental review of the relationship between nanotechnology and lignocellulosic biomass. In: Lucia LA, Rojas OJ, editors. The nanoscience and technology of renewable biomaterials, UK: Blackwell Publishing Ltd; 2009, p.1-42.

[2] Fernandes EM, Pires RA, Mano JF, Reis RL. Bionanocomposites from lignocellulosic resources: properties, applications and future trends for their use in the biomedical field. Prog Polym Sci 2013;38:1415-41.

[3] Lee K-Y, Aitomäki Y, Berglund LA, Oksman K, Bismarck A. On the use of nanocellulose as reinforcement in polymer matrix composites. Compos Sci Technol 2014;105:15-27.

[4] Israelachvili JN, Tabor D. Measurement of van der Waals dispersion forces in range 1.5 to 130 nm. Proc R Soc London Ser A: Math Phys Eng Sci 1972;331:19-38. 
[5] Israelachvili JN, Adams GE. Measurement of forces between two mica surfaces in aqueous electrolyte solutions in the range 0-100 nm. J Chem Soc Faraday Trans 1978;74:975-1001.

[6] Binnig G, Quate CF, Gerber C. Atomic force microscope. Phys Rev Lett 1986;56:930-3.

[7] Ducker WA, Senden TJ, Pashley RM. Direct measurement of colloidal forces using an atomic force microscope. Nature 1991;353:239-41.

[8•] Neuman RD, Berg JM, Claesson PM. Direct measurement of surface forces in papermaking and paper coating systems. Nord Pulp Paper Res J 1993;8:96-104. The first direct surface force measurements between cellulose surfaces.

[9••] Holmberg M, Berg J, Stemme S, Ödberg L, Rasmusson J, Claesson P. Surface force studies of Langmuir-Blodgett cellulose films. J Colloid Interface Sci 1997;186:369-81. Force measurements between well characterized cellulose surfaces prepared by Langmuir-Blodgett deposition. Estimation of Hamaker constant for cellulose across air and water.

[10] Bergström L, Stemme S, Dahlfors T, Arwin H, Ödberg L. Spectroscopic ellipsometry characterisation and estimation of the Hamaker constant of cellulose. Cellulose 1999;6:1-13.

[11•] Rutland MW, Carambassis A, Willing GA, Neuman RD. Surface force measurements between cellulose surfaces using scanning probe microscopy. Colloid Surf A-Physicochem Eng Asp 1997;123-124:369-74. First AFM colloidal probe measurements of forces between cellulose surfaces.

[12] Derjaguin BV, Landau L. Theory of the stability of strongly charged lyophobic sols and of the adhesion of strongly charged particles in solution of electrolytes. Acta Phys Chim USSR 1941;14:633-62.

[13] Verwey EJW, Overbeek JTG. Theory of the stability of lyophobic colloids. Amsterdam: Elsevier; 1948.

[14] Österberg M, Claesson PM. Interactions between cellulose surfaces: effect of solution pH. J Adhesion Sci Technol 2000;14:603-18.

[15••] Carambassis A, Rutland MW. Interactions of cellulose surfaces: effect of electrolyte. Langmuir 1999;15:5584-90. Effect of $\mathrm{pH}$ and ionic strength on the interactions between cellulose surfaces. Correlation to DLVO theory.

[16] Zauscher S, Klingenberg DJ. Normal forces between cellulose surfaces measured with colloidal probe microscopy. J Colloid Interface Sci 2000;229:497-510.

[17•] Notley SM, Pettersson B, Wågberg L. Direct measurement of attractive van der Waals’ forces between regenerated cellulose surfaces in an aqueous environment. J Am Chem Soc 2004;126:13930-1. First direct measurement of van der Waals attraction between cellulose surfaces. 
[18] Aulin C, Ahola S, Josefsson P, Nishino T, Hirose Y, Österberg, M, Wågberg L. Nanoscale cellulose films with different crystallinities and mesostructures - their surface properties and interaction with water. Langmuir 2009;25:7675-85.

[19] Kontturi E, Tammelin T, Österberg M. Cellulose - model films and the fundamental approach. Chem Soc Rev 2006;35:1287-304.

[20] Wågberg L, Österberg M, Enarsson L-E. Interactions at cellulose model surfaces. Encyclopedia of Surface and Colloid Science 2010; 1: 1, 1-19.

[21•] Notley SM. Effect of introduced charge in cellulose gels on surface interactions and the adsorption of highly charged cationic polyelectrolytes. Phys Chem Chem Phys 2008;10:181925. Study on how the surface charge affects the interaction between cellulose surfaces.

[22••] Ahola S, Salmi J, Johansson L-S, Laine J, Österberg M. Model films from native cellulose nanofibrils. Preparation, swelling, and surface interactions. Biomacromolecules 2008;9:127382. Preparation and characterization of CNF films for the first time.

[23] Krässig HA. Effect of structure and morphology on accessibility and reactivity. In: Krässig HA, editor. Cellulose: structure, accessibility and reactivity, The Netherlands: Gordon and Breach Science Publishers; 1993, vol.11, p.167-324.

[24•] Notley SM, Eriksson M, Wågberg L, Beck S, Gray DG. Surface forces measurements of spincoated cellulose thin films with different crystallinity. Langmuir 2006;22:3154-60. Comparison of surface forces between different cellulose substrates in various electrolyte media.

[25•] Stiernstedt J, Nordgren N, Wågberg L, Brumer H, Gray DG, Rutland MW. Friction and forces between cellulose model surfaces: a comparison. J Colloid Interface Sci 2006;303:117-23. Effect of roughness on friction forces between cellulose surfaces.

[26] Hubbe MA, Nanko H, McNeal MR. Retention aid polymer interactions with cellulosic surfaces and suspensions: a review. Bioresources 2009;4:850-906.

[27•] Österberg M. The effect of a cationic polyelectrolyte on the forces between two cellulose surfaces and between one cellulose and one mineral surface. J Colloid Interface Sci 2000;229:620-7. Effect of the adsorbed amount of cationic polyelectrolyte on the interaction between cellulose and mineral surfaces.

[28] Holmberg M, Wigren R, Erlandsson R, Claesson PM. Interactions between cellulose and colloidal silica in the presence of polyelectrolytes. Colloid Surf A-Physicochem Eng Asp 1997;129-130:175-83.

[29] Radtchenko IL, Papastavrou G, Borkovec M. Direct force measurements between cellulose surfaces and colloidal silica particles. Biomacromolecules 2005;6:3057-66. 
[30] Parker JL. A novel method for measuring the force between two surfaces in a surface force apparatus. Langmuir 1992;8:551-6.

[31] Poptoshev E, Rutland MW, Claesson PM. Surface forces in aqueous polyvinylamine solutions. 2. Interactions between glass and cellulose. Langmuir 2000;16:1987-92.

[32] Leporatti S, Sczech R, Riegler H, Bruzzano S, Storsberg J, Loth F, Jaeger W, Laschewsky A, Eichhorn S, Donath E. Interaction forces between cellulose microspheres and ultrathin cellulose films monitored by colloidal probe microscopy - effect of wet strength agents. J Colloid Interface Sci 2005;281:101-11.

[33] Notley SM, Chen W, Pelton R. Extraordinary adhesion of phenylboronic acid derivatives of polyvinylamine to wet cellulose: a colloidal probe microscopy investigation. Langmuir 2009;25:6898-904.

[34•] Salmi J, Österberg M, Stenius P, Laine J. Surface forces between cellulose surfaces in cationic polyelectrolyte solutions: the effect of polymer molecular weight and charge density. Nord Pulp Paper Res J 2007;22:249-57. Analysis of how the molecular weight and charge of cationic polyelectrolytes affect the interaction between cellulose surfaces.

[35••] Salmi J, Österberg M, Laine J. The effect of cationic polyelectrolyte complexes on interactions between cellulose surfaces. Colloid Surf A-Physicochem Eng Asp 2007;297:12230. Unique work where the interaction between cellulose surfaces were studied in the presence of polyelectrolyte complexes.

[36] Amelina EA, Shchukin ED, Parfenova AM, Bessonov AI, Vidensky IV. Adhesion of the cellulose fibers in liquid media: 1. Measurement of the contact friction force. Colloid $\mathrm{J}$ 1998;60:537-40.

[37] Amelina EA, Shchukin ED, Parfenova AM, Pelekh VV, Vidensky IV, Bessonov AI, Aranovich G, Donohue M. Effect of cationic polyelectrolyte and surfactant on cohesion and friction in contacts between cellulose fibers. Colloid Surf A-Physicochem Eng Asp 2000;167:215-27.

[38] Amelina et al. Contact interactions between individual fibers of cellulose and its derivatives: mechanism of cationic surfactant action. Colloid J 2001;63:527-31.

[39] Huang F, Li K, Kulachenko A. Measurement of interfiber friction force for pulp fibers by atomic force microscopy. J Mater Sci 2009;44:3770-6.

[40•] Feiler AA, Stiernstedt J, Theander K, Jenkins P, Rutland MW. Effect of capillary condensation on friction force and adhesion. Langmuir 2007;23:517-22. Study of the effect of the relative humidity on the adhesion and friction between cellulose surfaces. 
[41] Garoff N, Zauscher S. The influence of fatty acids and humidity on friction and adhesion of hydrophilic polymer surfaces. Langmuir 2002;18:6921-7.

[42] Bogdanovic G, Tiberg F, Rutland MW. Sliding friction between cellulose and silica surfaces. Langmuir 2001;17:5911-6.

[43•] Zauscher S, Klingenberg DJ. Surface and friction forces between cellulose surfaces measured with colloidal probe microscopy. Nord Pulp Paper Res J 2000;15:459-68. Normal and friction forces between cellulose surfaces in the presence of various polyelectrolytes relevant for papermaking.

[44] Theander K, Pugh RJ, Rutland MW. Friction force measurements relevant to de-inking by means of atomic force microscope. J Colloid Interface Sci 2005;291:361-8.

[45••] Theander K, Pugh RJ, Rutland MW. Forces and friction between hydrophilic and hydrophobic surfaces: influence of oleate species. J Colloid Interface Sci 2007;313:735-46. Surface and friction force measurements relevant for deinking process.

[46••] Notley SM, Norgren M. Measurement of interaction forces between lignin and cellulose as a function of aqueous electrolyte solution conditions. Langmuir 2006;22:11199-204. Measurement of the interaction between cellulose and lignin.

[47] Österberg M, Laine J, Stenius P, Kumpulainen A, Claesson PM. Forces between xylan-coated surfaces: effect of polymer charge density and background electrolyte. J Colloid Interface Sci 2001;242:59-66.

[48•] Paananen A, Österberg M, Rutland MW, Tammelin T, Saarinen T, Tappura K, Stenius P. Interaction between cellulose and xylan: an atomic force microscope and quartz crystal microbalance study. ACS Symp Ser 2004;864:269-90. Effect of xylan adsorption on cellulose interactions.

[49••] Stiernstedt J, Brumer H, Zhou Q, Teeri TT, Rutland MW. Friction between cellulose surfaces and effect of xyloglucan adsorption. Biomacromolecules 2006;7:2147-53. Effect of xyloglucan on surface and friction forces between cellulose surfaces.

[50] Nordgren N, Eklöf J, Zhou Q, Brumer H, Rutland MW. Top-down grafting of xyloglucan to gold monitored by QCM-D and AFM: enzymatic activity and interactions with cellulose. Biomacromolecules 2008;9:942-8.

[51] Nordgren N, Eronen P, Österberg M, Laine J, Rutland MW. Mediation of the nanotribological properties of cellulose by chitosan adsorption. Biomacromolecules 2009;10:645-50.

[52] Klemm D, Kramer F, Moritz S, Lindström T, Ankerfors M, Gray D, Dorris A. Nanocelluloses: a new family of nature-based materials. Angew Chem Int Ed 2011;50:5438-66. 
[53] Nigmatullin R, Lovitt R, Wright C, Linder M, Nakari-Setälä T, Gama M. Atomic force microscopy study of cellulose surface interaction controlled by cellulose binding domains. Colloid Surf B-Biointerfaces 2004;35:125-35.

[54] Qin C, Clarke K, Li K. Interactive forces between lignin and cellulase as determined by atomic force microscopy. Biotechnol Biofuels 2014;7:65.

[55] Arslan B, Colpan M, Ju X, Zhang X, Kostyukova A, Abu-Lail NI. The effects of noncellulosic compounds on the nanoscale interaction forces measured between carbohydrate-binding module and lignocellulosic biomass. Biomacromolecules 2016;17:1705-15.

[56] Salmi J, Nypelö T, Österberg M, Laine J. Layer structures formed by silica nanoparticles and cellulose nanofibrils with cationic polyacrylamide (C-PAM) on cellulose surface and their influence on interactions. BioResources 2009;4:602-25.

[57] Aulin C, Johansson E, Wågberg L, Lindström T. Self-organized films from cellulose I nanofibrils using the layer-by-layer technique. Biomacromolecules 2010;11:872-82.

[58••] Eronen P, Laine J, Ruokolainen J, Österberg M. Comparison of multilayer formation between different cellulose nanofibrils and cationic polymers. J Colloid Interface Sci 2012;373:84-93. In situ force measurements during the formation of multilayers containing anionic and cationic CNF.

[59] Olszewska AM, Kontturi E, Laine J, Österberg M. All-cellulose multilayers: long nanofibrils assembled with short nanocrystals. Cellulose 2013;20:1777-89.

[60••] Cranston ED, Gray DG, Rutland MW. Direct surface force measurements of polyelectrolyte multilayer films containing nanocrystalline cellulose. Langmuir 2010;26:17190-7. Interesting proof of migration of polymers through $\mathrm{CNC}$ layer in the interactions between preformed polyelectrolyte multilayers containing $\mathrm{CNC}$ and a colloidal probe.

[61] Benítez AJ, Lossada F, Zhu B, Rudolph T, Walther A. Understanding toughness in bioinspired cellulose nanofibril/polymer nanocomposites. Biomacromolecules 2016;17:2417-26.

[62] Buehler MJ. Nature designs tough collagen: explaining the nanostructure of collagen fibrils. Proc Natl Acad Sci USA 2006;103:12285-90.

[63] Launey ME, Buehler MJ, Ritchie RO. On the mechanistic origins of toughness in bone. Annu Rev Mater Res 2010;40:25-53.

[64] Palmeri MJ, Putz KW, Brinson LC. Sacrificial bonds in stacked-cup carbon nanofibers: biomimetic toughening mechanisms for composite systems. ACS Nano 2010;4:4256-64.

[65] Sen D, Buehler MJ. Structural hierarchies define toughness and defect-tolerance despite simple and mechanically inferior brittle building blocks. Sci Rep 2011;1:35. 
[66••] Olszewska A, Junka K, Nordgren N, Laine J, Rutland MW, Österberg M. Non-ionic assembly of nanofibrillated cellulose and polyethylene glycol grafted carboxymethyl cellulose and the effect of aqueous lubrication in nanocomposite formation. Soft Matter 2013;9:7448-57. A first attempt to apply direct surface force measurements to explain properties of biomimetic nanocomposites.

[67•] Olszewska A, Valle-Delgado JJ, Nikinmaa M, Laine J, Österberg M. Direct measurements of non-ionic attraction and nanoscaled lubrication in biomimetic composites from nanofibrillated cellulose and modified carboxymethylated cellulose. Nanoscale 2013;5:11837-44. Adhesion and friction forces in air between nanocellulose surfaces and CMC-PEG. Correlation to mechanical properties of composites.

[68] Tang H, Butchosa N, Zhou Q. A transparent, hazy, and strong macroscopic ribbon of oriented cellulose nanofibrils bearing poly(ethylene glycol). Adv Mater 2015;27:2070-6.

[69] Lahiji RR, Boluk Y, McDermott M. Adhesive surface interactions of cellulose nanocrystals from different sources. J Mater Sci 2012;47:3961-70.

[70] Nordgren N, Lönnberg H, Hult A, Malmström E, Rutland MW. Adhesion dynamics for cellulose nanocomposites. ACS Appl Mater Interfaces 2009;1:2098-103.

[71] Guan Y, Xiao H, Sullivan H, Zheng A. Antimicrobial-modified sulfite pulps prepared by in situ copolymerization. Carbohydr Polym 2007;69:688-96.

[72] Zoppe JO, Österberg M, Venditti RA, Laine J, Rojas OJ. Surface interaction forces of cellulose nanocrystals grafted with thermoresponsive polymer brushes. Biomacromolecules 2011;12:2788-96.

[73] Greene GW, Olszewska A, Österberg M, Zhu H, Horn R. A cartilage-inspired lubrication system. Soft Matter 2014;10:374-82.

[74•] Valle-Delgado JJ, Johansson L-S, Österberg M. Bioinspired lubricating films of cellulose nanofibrils and hyaluronic acid. Colloids Surf B-Biointerfaces 2016;138:86-93. Very good lubrication achieved by grafting hyaluronic acid to CNF films. 


\section{Figure captions}

Figure 1. Normalized force curves between two cellulose spheres in solutions of $0.1 \mathrm{mM}(\$)$ and $10 \mathrm{mM} \mathrm{KBr}(f)$. The solid and dashed lines correspond to DLVO fits for double layer interaction at constant surface charge and constant surface potential, respectively. [Reprinted with permission from Carambassis and Rutland, Langmuir 1999;15:5584-90, Copyright 1999 American Chemical Society.]

Figure 2. Effect of cationic polyelectrolyte concentration on the surface forces between approaching cellulose surfaces in aqueous solutions: a) PDADMAC (intermediate molecular weight and high charge); b) C-PAM (high molecular weight, low charge). [Modified from reference [34•].]

Figure 3. Effect of sodium oleate concentration on the friction forces between a cellulose microsphere and an alkyd resin substrate in $1 \mathrm{mM} \mathrm{NaCl}$ solution at $\mathrm{pH} \mathrm{10.} \mathrm{(ò} \mathrm{,}{ }^{\circ}$ ) denotes surfactant free solution, and $(\varnothing, f),(\varnothing, \varnothing),(p, r)$ and $(q, s)$ correspond to concentrations of 0.015, 0.1, 1 and $10 \mathrm{cmc}$, respectively (closed and open symbols for loading and unloading, respectively). [Reprinted with permission from Theander et al., J Colloid Interface Sci 2007;313:735-46, Copyright 2007 Elsevier.]

Figure 4. Normalized force curves between surfaces on approach during multilayer build up: a) multilayer of PDADMAC and highly negatively charged CNF (ref.= CNF; 1.= PDADMAC; $2 .=$ CNF; 3.= PDADMAC); b) multilayer of cationic CNF and highly negatively charged CNF (ref.= CNF; 1.= cationic CNF; 2.= CNF; 3.= cationic CNF; 4.= CNF). The solid line shows the contribution of double layer forces. The inset shows the corresponding steps of layer-by-layer deposition from QCM-D measurements. [Reprinted with permission from Eronen et al., J Colloid Interface Sci 2012;373:84-93, Copyright 2012 Elsevier.]

Figure 5. Normalized force curves for the interaction between a silica colloid probe and a $\mathrm{PAH} / \mathrm{NCC}$ multilayer in aqueous solution at $\mathrm{pH} 3.5$ and different ionic strengths. Inset shows a schematic representation of the PAH chains extending and bridging more as the ionic strength increases (NCC: blue rods; PAH: green lines). [Reprinted with permission from Cranston et al., Langmuir 2010;26:17190-7, Copyright 2010 American Chemical Society.] 
Figure 6. Effect of $\mathrm{pH}$ and ionic strength on the surface forces (a) and friction forces (b) between a glass microsphere and a CNF film with hyaluronic acid attached. Closed and open symbols in (b) correspond to loading and unloading experiments, respectively. PBS: phosphate buffered saline (high ionic strength); PB: phosphate buffer (low ionic strength). [Reprinted with permission from Valle-Delgado et al., Colloids Surf B-Biointerfaces 2016;138:86-93, Copyright 2016 Elsevier.] 
Figure 1

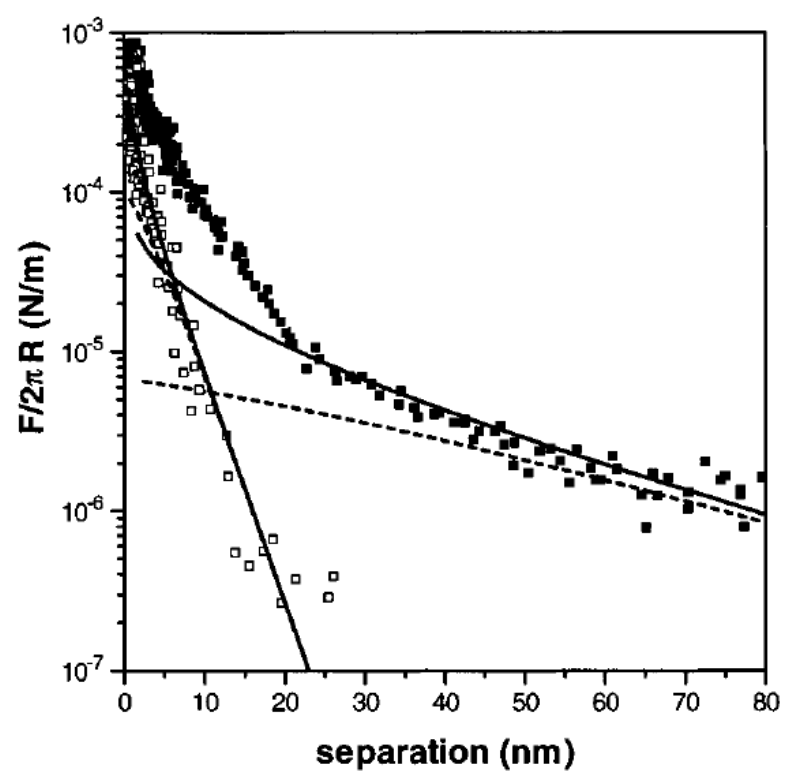


Figure 2

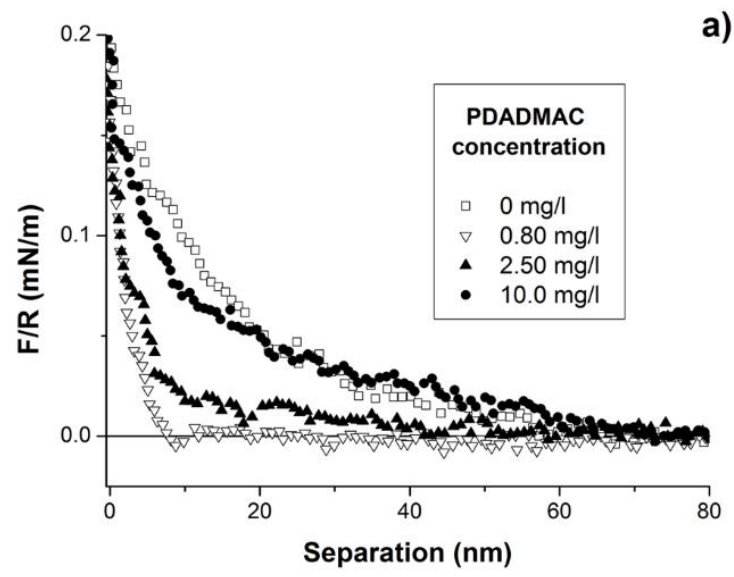

a)

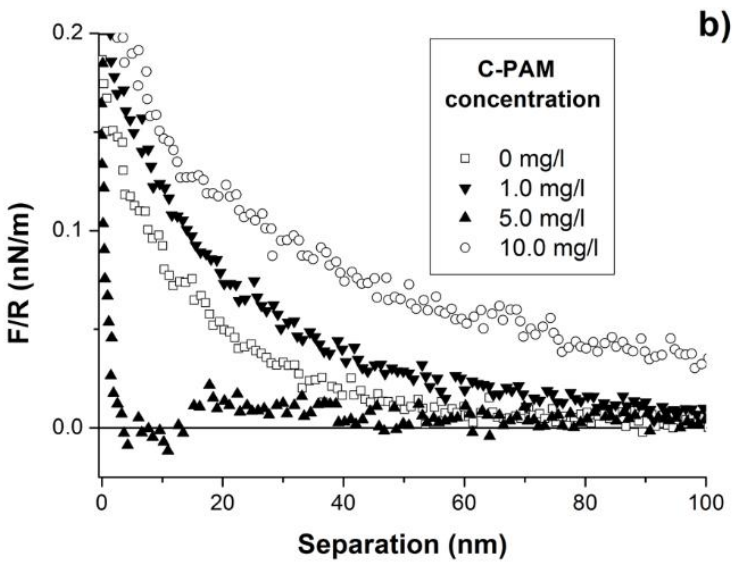


Figure 3

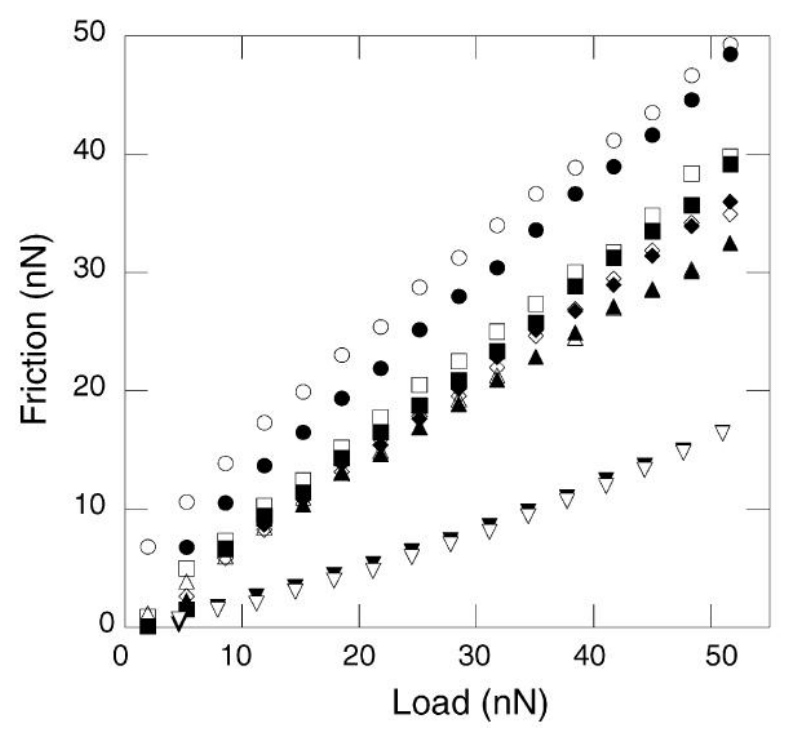


Figure 4

a)
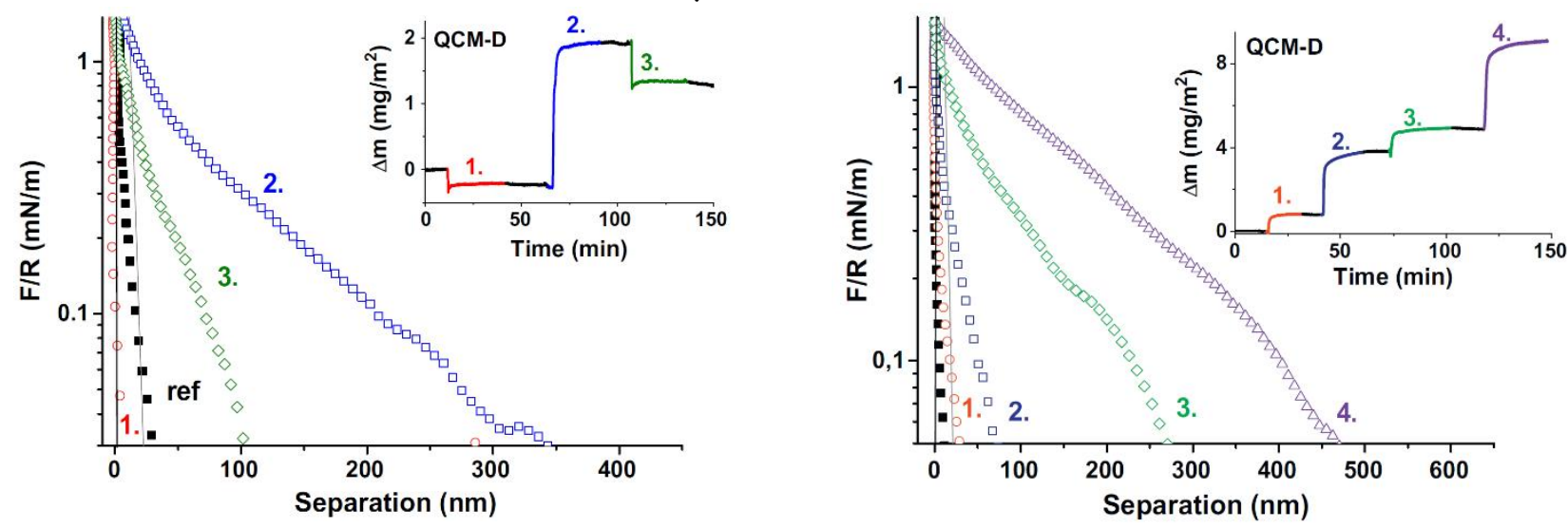

b) 
Figure 5

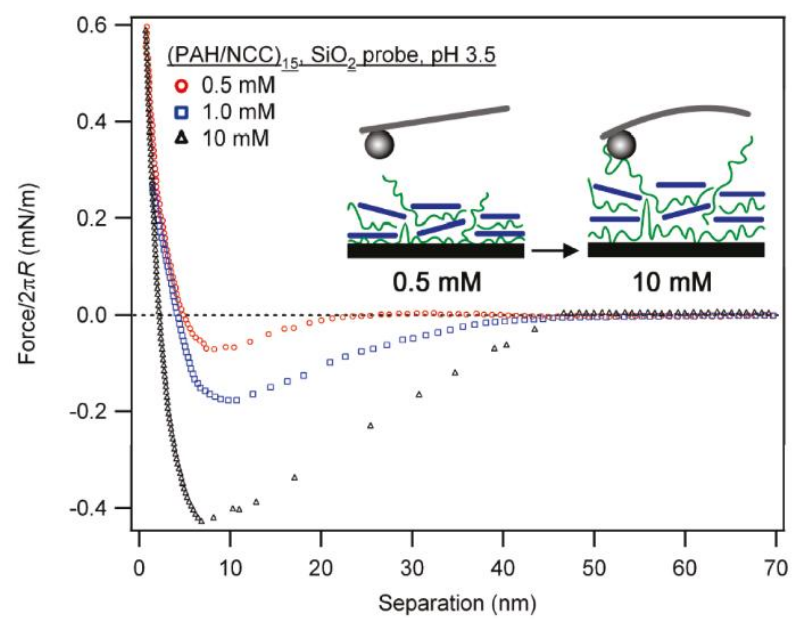


Figure 6
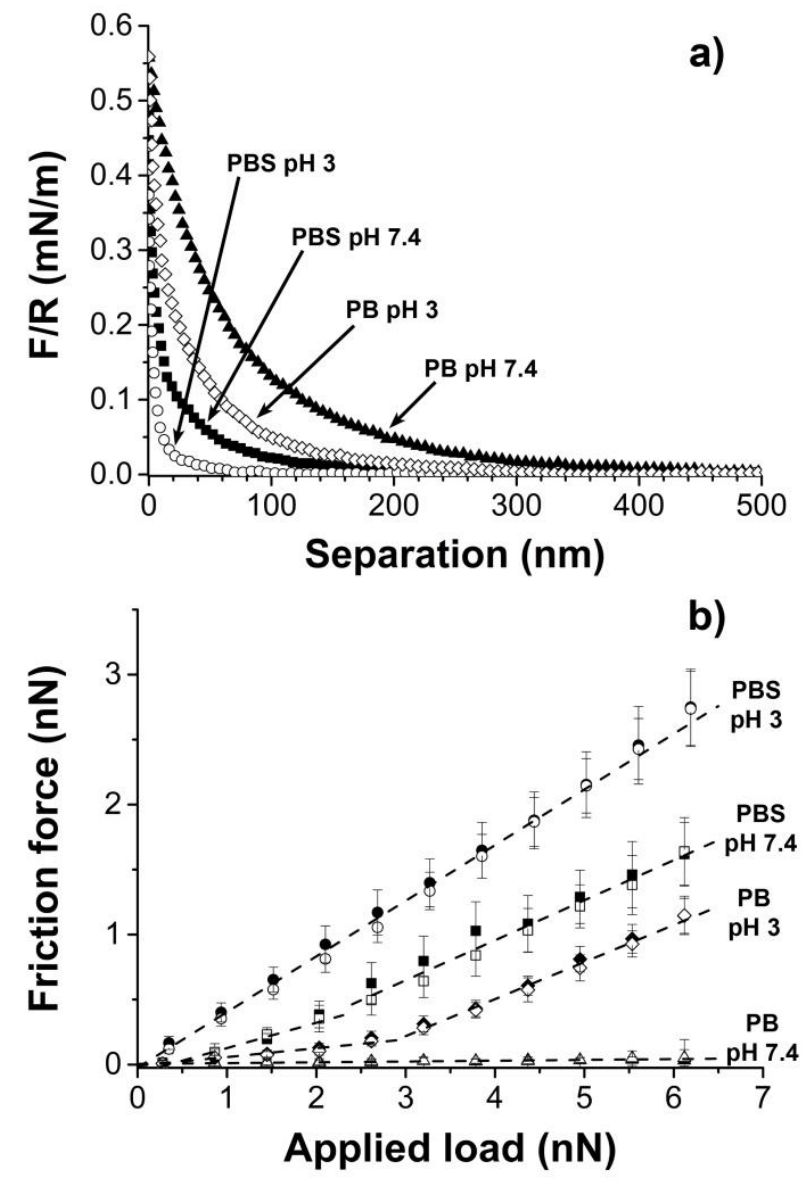
Graphical Abstract

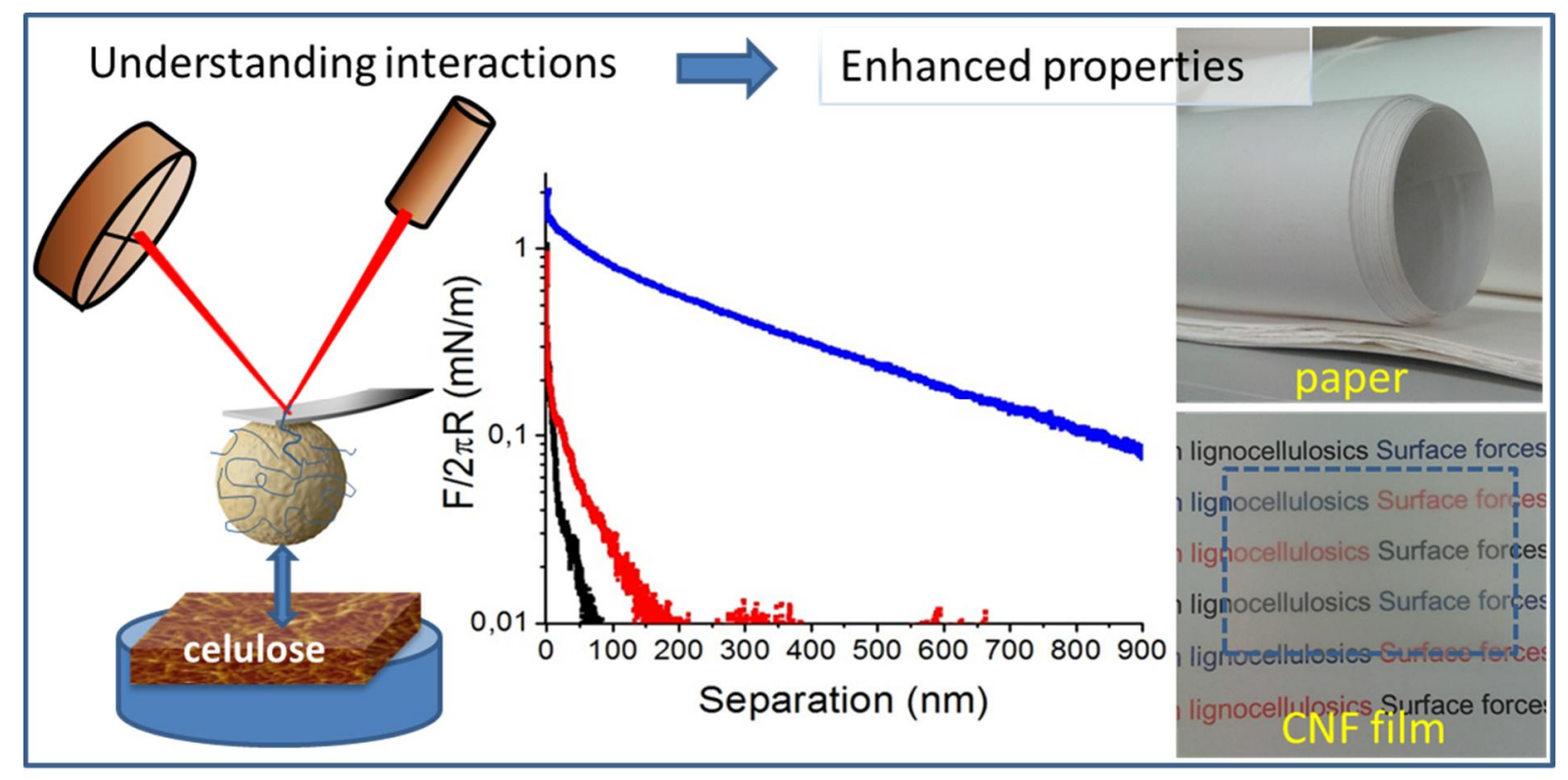

